\title{
Modified Buck-Boost Converter as DC Motor Driver with Continuous Input Current
}

\author{
FELIX A. HIMMELSTOSS ${ }^{1} \&$ KARL EDELMOSER ${ }^{2}$ \\ ${ }^{1}$ Power Electronics Department \\ University of Applied Science Technikum Wien \\ Hoechstaedtplatz 6, 1200 Wien \\ ${ }^{2}$ Institute of Energy Systems and Electrical Drives \\ University of Technology Vienna \\ Gusshausstraße 24-26, 1040 Wien \\ AUSTRIA
}

\begin{abstract}
A modified Buck-Boost converter with output filter is treated as a switched mode power supply and as a two quadrant driver for DC machines. The modification is done by changing the position of the capacitor of the Buck-Boost. With this modification the input and the output currents of the converter are steady and the maximum input current is reduced compared to a normal Buck-Boost. The function of the modified converter is explained with the help of time signals, the large signal and the small signal models are derived, the transfer functions for the speed are calculated, dimensioning hints are given, and simulations are shown.
\end{abstract}

Key-Words: - DC/DC converter, DC motor drive, two-quadrant, modified Buck-Boost, modelling, large and small signal model, transfer function

Received: February 19, 2020. Revised: August 13, 2020. Accepted: August 30, 2020. Published: September 23, 2020

\section{Introduction}

$\mathrm{DC} / \mathrm{DC}$ converters transfer an input voltage $\mathrm{U}_{1}$ into an output voltage $U_{2}$. They are described at length in the text books $[1,2,3]$. In conference and journal papers further converter topologies are published. A modified Buck-Boost converter [4] is treated here. The position of the output capacitor of a classical Buck-Boost converter is changed. It is now connected between the input and the output. This leads to a constant input current. Furthermore, to achieve a continuous output current, an output filter is added. If a DC machine is connected instead of the filter, a motor driving device is achieved. DC motors are produced and used in many millions pieces every year. But no new concepts for drivers are published in the literature, only some aspects of the control were treated recently $[6,7]$. In this paper we show and discuss a new converter topology with constant input and output current for this aim.

\subsection{Derivation of the converter topology}

Starting from the Buck-Boost converter with output filter in Fig. 1. we change the position of the capacitor $\mathrm{C}_{1}$ (Fig. 2).

The converter consists of two input connectors for applying the input voltage $\mathrm{U}_{1}$, an active switch $\mathrm{S}$, which is controlled by the signal $\mathrm{G}$, an inductor $\mathrm{L}_{1}$, a diode $\mathrm{D}$, and a capacitor $\mathrm{C}_{1}$. The output filter is formed by the inductor $\mathrm{L}_{2}$ and the capacitor $\mathrm{C}_{2}$. The load is rep

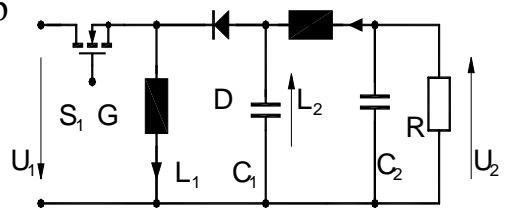

Fig. 1. Buck-Boost converter with output filter

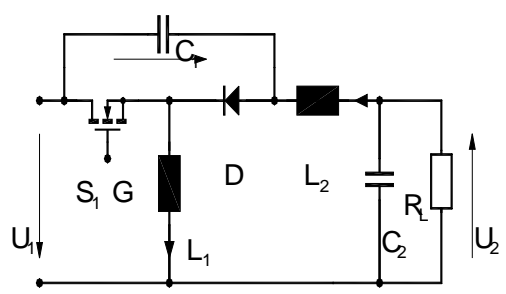

Fig. 2. Modified Buck-Boost converter with output filter

\subsection{Derivation of the converter topology}

To get a better idea of the converter we design some important signals in the steady state mode. Ideal elements are assumed. With a duty cycle of $2 / 3$ one can draw the cc

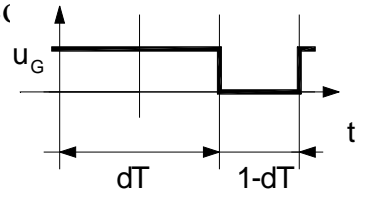

Fig. 3. Control signal 
To obtain the voltage transformation rate, one has to inspect the voltages across the inductive elements. In the steady-state they must be zero in the mean. One can draw the voltage across the inductors (Fig. 4).

The voltage across $\mathrm{L}_{2}$ is always built by a mesh consisting of the input voltage and the voltages across the capacitors. Therefore, the voltage is always zero and one gets a constant current through the output inductor. This is a very interesting feature of the modified converter with output filter. The current through the filter inductor is therefore nearly constant.
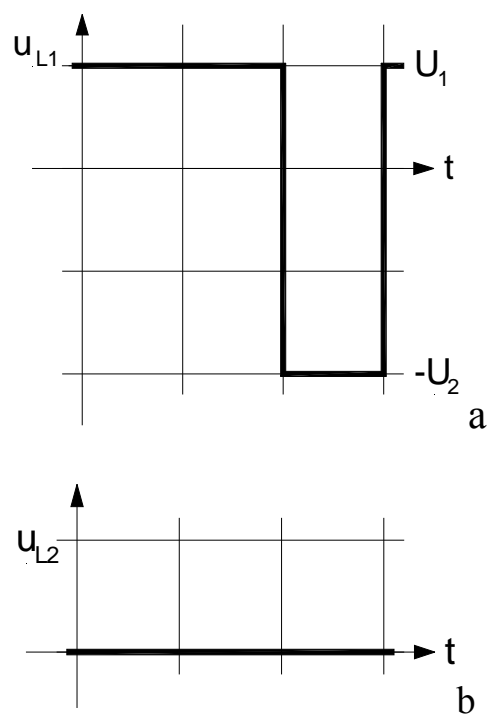

Fig. 4. Voltages across the inductors

As the voltage across an inductor must be zero in the mean, the positive and the negative voltage-time areas must be the same. With this voltage-time balance, the voltage transformation rate can be achieved. The modified converter has the same voltage transformation rate between the output voltage and the input voltage as the well-known Buck-Boost

$$
M=\frac{U_{2}}{U_{1}}=\frac{d}{1-d} .
$$

\subsection{Currents in the converter}

To get the connection between the currents, one has to inspect the current through the capacitors. They have to be zero in the mean. For $\mathrm{C}_{1}$ one can draw the current according to Fig. 5.

The charge balance

$$
\bar{I}_{L 2} \cdot d=\left(\bar{I}_{L 1}-\bar{I}_{L 2}\right)(1-d)
$$

leads to

$$
\bar{I}_{L 1}=\frac{\bar{I}_{L 2}}{1-d} .
$$

The voltage across $L_{1}$ must have a current ripple shown in Fig. 6.

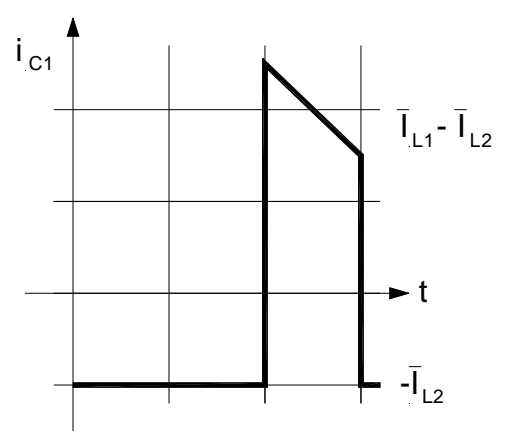

Fig. 5. Current through $\mathrm{C}_{1}$

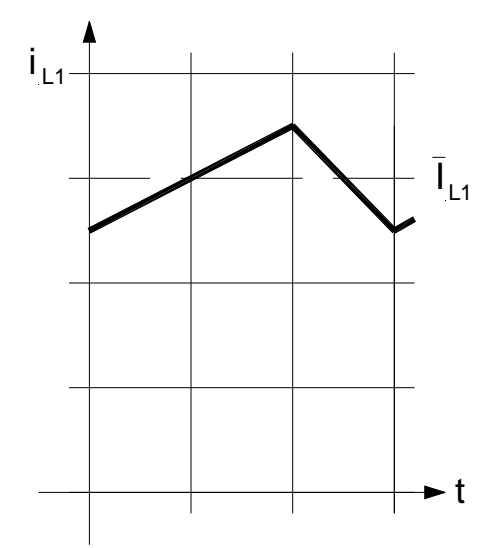

Fig. 6. Current through the inductor $\mathrm{L}_{1}$

The voltage across $L_{2}$ is nearly constant zero all the time and therefore the current through the second inductor is practically constant. The current is equal to the load current Fig. 7. Therefore, only a small filter inductor can be used.

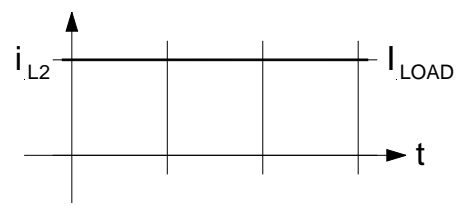

Fig. 7. Current through inductor $\mathrm{L}_{2}$

Now we consider another interesting aspect of this modified converter. The input current of the converter is always the difference between the inductor currents, whether the active switch $\mathrm{S}$ or the diode $\mathrm{D}$ is conducting

$$
i_{I N}=i_{L 1}-i_{L 2} \text {. }
$$

The input current is therefore steady (Fig.8). Comparing it with the input current of the BuckBoost converter with output filter one gets Fig. 9.

The input current of the normal Buck-Boost is pulsating and the maximum value is higher. The input current has therefore a higher amplitude. 


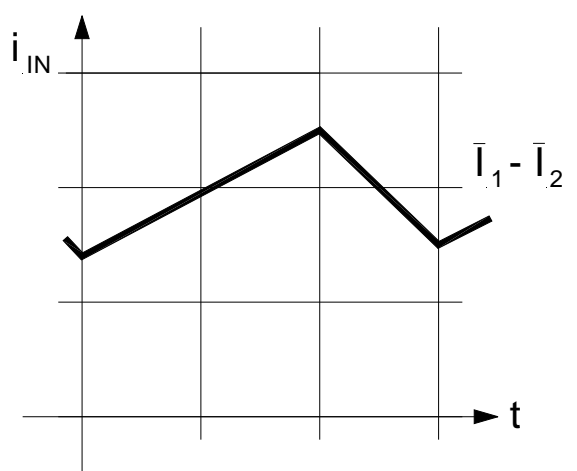

Fig. 8. Input current of the modified converter

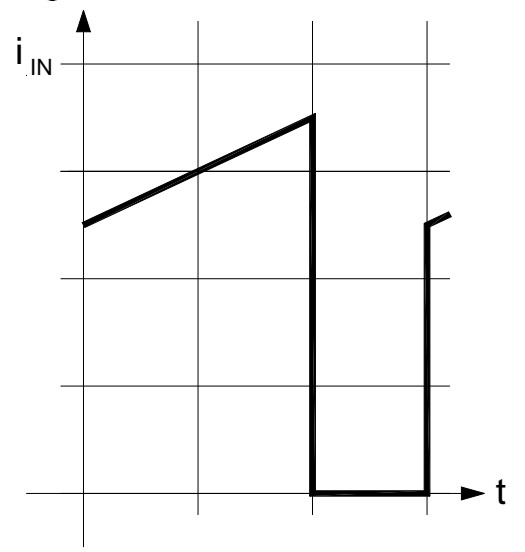

Fig. 9. Input current of the normal Buck-Boost converter with output filter

A second interesting aspect of the modified converter is that the voltage across $C_{1}$ is always the sum of the input voltage and the output voltage. The energy stored in this capacitor is therefore higher than in the classical version of the Buck-Boost. This can be an advantage during transients or one can use a smaller capacitor.

\subsection{Loss reduction}

To reduce the losses, the diode is replaced by an active switch as shown in Fig. 10.

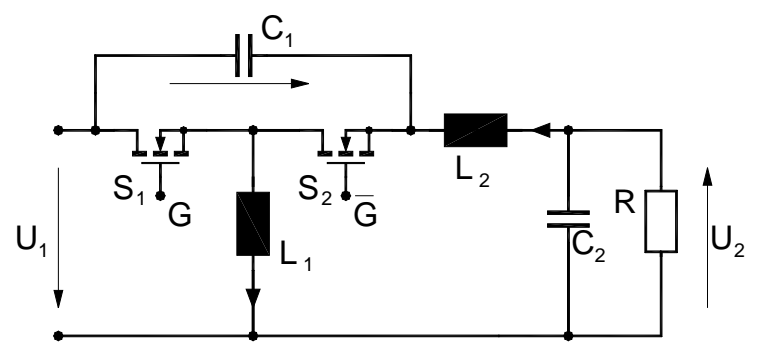

Fig. 10. Loss reduction by active rectification

The body diodes of the MOSFETs are necessary in this case. When $S_{1}$ is turned off, the body diode of $\mathrm{S}_{2}$ turns on and the current through $\mathrm{L}_{1}$ commutates into it. Now $\mathrm{S}_{2}$ is turned on shunting the diode and the current flows through the channel of the MOSFET $S_{2}$. When $S_{2}$ is turned off, the current commutates into the body diode again. Now $\mathrm{S}_{1}$ can be turned on again and the current commutates into the channel of $S_{1}$. To avoid a short circuit, there has always to be a dead time between the control signals of the switches.

Fig. 11 shows the modified half-bridge Buck-Boost converter with explicitly drawn diodes. These diodes are also necessary when e.g. IGBTs are used for higher voltages and powers.

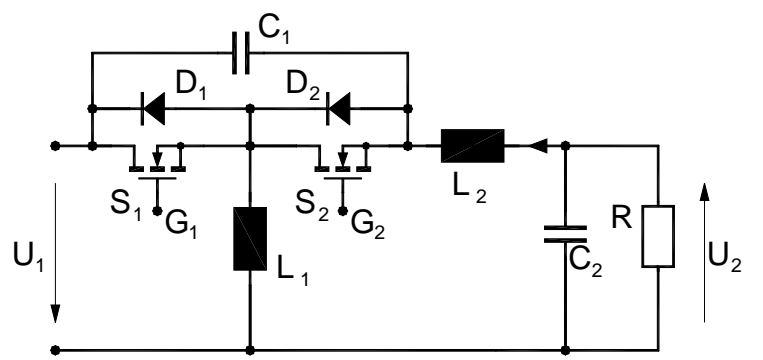

Fig. 11. Modified Buck-Boost converter with explicitly drawn diodes

\section{Modified half-bridge Buck-Boost converter as motor driver}

The modified Buck-Boost converter with active rectification can be used as a two quadrant driver for a permanent exited DC machine (Fig. 12). In this case $\mathrm{L}_{2}$ is the armature inductor and the voltage across the capacitor describes the source voltage of the machine. The momentum of inertia $J$ can be described by the value of the capacitor and the load can be interpreted as a resistor or even better by a controlled extern current source instead of the resistor. When IGBTs are used instead of MOSFETs, external diodes are necessary.

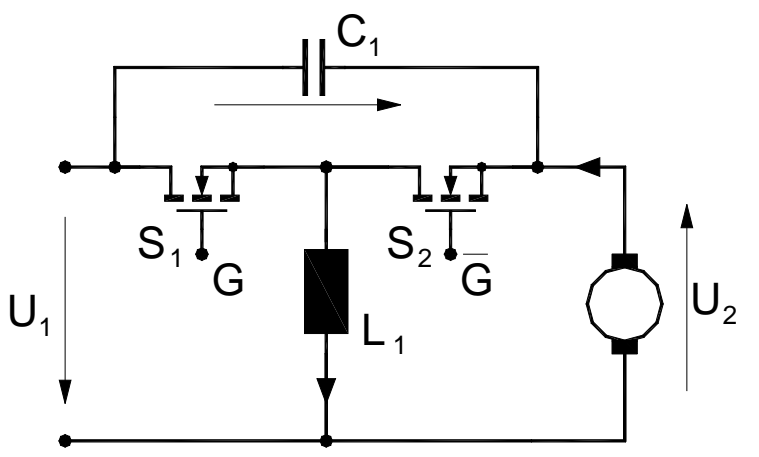

Fig. 12. Modified Buck-Boost as two-quadrant motor driver

\subsection{Model of the modified Buck-Boost converter}

The model of the machine consists of an inductor $L_{M}$, a resistor $R_{M}$ and a source voltage, which is dependent on the speed $\mathrm{n}$. With the voltage-constant of the machine $C_{E}$, the voltage across the terminals $\mathrm{u}_{\mathrm{M}}$ can be described by

$$
u_{M}=R_{M} i_{M}+L_{M} \frac{d i_{M}}{d t}+C_{E} n .
$$


In the ideal case, all parasitic resistances (including $\mathrm{R}_{\mathrm{M}}$ ) are set to zero, the stationary speed can now be calculated according to

$$
n=\frac{1}{C_{E}} \frac{d}{1-d} U_{1} .
$$

In the continuous inductor current mode, the converter has two modes depending on which transistor is conducting. During mode M1 the first active switch $\mathrm{S}_{1}$ is conducting and the second switch $\mathrm{S}_{2}$ is blocking, and in mode M2 it is the other way around. The complete drive, consisting of the modified converter and the DC machine, can be described by four state variables: the current in the inductor of the converter $i_{L 1}$, the current through the machine $i_{M}$, the voltage across the capacitor of the converter $\mathrm{u}_{\mathrm{Cl}}$, and the rotating speed $\mathrm{n}$. There are also two input variables: the input voltage $\mathrm{u}_{1}$ and the momentum of the load $\mathrm{m}_{\mathrm{L}}$. The parasitic resistances are taken into account. $\mathrm{R}_{\mathrm{L} 1}$ is the resistor of the inductor and $\mathrm{R}_{\mathrm{Cl}}$ the resistor of the capacitor of the converter, $\mathrm{R}_{\mathrm{S}}$ represents the on-resistor of the switches $S_{1}$ and $S_{2}$.

\subsubsection{First state equation}

The first state equation is the same as for a power supply according to Fig. 2.

For M1 one gets

$$
\frac{d i_{L 1}}{d t}=\frac{-R_{L 1} i_{L 1}-R_{S} i_{L 1}+u_{1}}{L_{1}}
$$

and for M2

$$
\frac{d i_{L 1}}{d t}=\frac{-R_{L 1} i_{L 1}-R_{S} i_{L 1}+R_{C 1}\left(i_{L 2}-i_{L 1}\right)-u_{C 1}+u_{1}}{L_{1}}
$$

\subsubsection{Second state equation}

The differential equation for the armature current can be written according to Kirchhoff's voltage law as:

For M1

$$
\frac{d i_{L M}}{d t}=\frac{-R_{M} i_{M}-C_{E} n-u_{1}+u_{C 1}-R_{C 1} i_{M}}{L_{M}}
$$

and for M2

$$
\frac{d i_{L M}}{d t}=\frac{-R_{M} i_{M}-C_{E} n-u_{1}+u_{C 1}+R_{C 1}\left(i_{L 1}-i_{M}\right)}{L_{M}} .
$$

\subsubsection{Third state equation}

The differential equation for the voltage across the capacitor can be written according to Kirchhoff's current law for the two modes as:

M1

M2 $\frac{d u_{C 1}}{d t}=\frac{i_{L 1}-i_{L M}}{C_{1}}$

$$
\frac{d u_{C 1}}{d t}=\frac{-i_{L M}}{C_{1}}
$$

\subsubsection{Fourth state equation}

The fourth state equation is Newton's axiom for rotation

$$
m_{B}=m_{M}-m_{\text {Load }}=J \frac{d \omega}{d t} .
$$

The produced momentum of the machine is proportional to the armature current. With the torque-constant $\mathrm{C}_{\mathrm{T}}$, the momentum of inertia $\mathrm{J}$ and using the speed $\mathrm{n}$ instead of the angular speed $\omega$ one can write

$$
\frac{d n}{d t}=\frac{C_{T} i_{M}-m_{\text {Load }}}{2 \pi J} .
$$

\subsection{Large signal model}

On the premises that the period of the switching frequency is much smaller than the time constants of the converter, the two sets of equations for the two modes can be combined and one gets the state-space description of the converter in the mean. The results of this model give the mean values of the state variables. The ripple cannot be seen in this model The ripple of the armature inductor, of the capacitor voltage, and of the speed can be neglected due to the large time constants for these variables. Only the current through the converter coil $\mathrm{L}_{1}$ is about $20 \%$, depending on the chosen inductor value, but on the dynamic analyses this has no influence. The statespace description is therefore

$\frac{d}{d t}\left(\begin{array}{c}i_{L 1} \\ i_{M} \\ u_{C 1} \\ n\end{array}\right)=$

$$
\begin{gathered}
{\left[\begin{array}{cccc}
-\frac{R_{C 1}(1-d)+R_{L 1}+R_{S}}{L_{1}} & \frac{R_{C 1}(1-d)}{L_{1}} & -\frac{1-d}{L_{1}} & 0 \\
\frac{R_{C 1}(1-d)}{L_{M}} & -\frac{R_{C 1}+R_{M}}{L_{M}} & \frac{1}{L_{M}} & -\frac{C_{E}}{L_{M}} \\
\frac{1-d}{C_{1}} & -\frac{1}{C_{1}} & 0 & 0 \\
0 & \frac{C_{T}}{2 \pi J} & 0 & 0
\end{array}\right]} \\
\left(\begin{array}{c}
i_{L 1} \\
i_{M} \\
u_{C 1} \\
n
\end{array}\right)+\left[\begin{array}{cc}
\frac{1}{L_{1}} & 0 \\
-\frac{1}{L_{M}} & 0 \\
0 & 0 \\
0 & -\frac{1}{2 \pi J}
\end{array}\right]\left(\begin{array}{c}
u_{1} \\
m_{\text {Load }}
\end{array}\right)
\end{gathered}
$$

This equation can be called the large signal model, because no restrictions for the variables are necessary (except the input voltage must be positive). All resistive parasitic elements are included in this model. The most influencing one is 
the armature resistor. Compared to this resistor the others parasitic resistors are very small.

\section{Linearization}

The large signal model is a nonlinear one, caused by the multiplications between two variables (e.g. between the duty cycle and the currents through $\mathrm{L}_{1}$ or $\mathrm{L}_{\mathrm{M}}$ ). To get the transfer functions of the drive, one has to linearize the equation (15) around a working point. A method to linearize the largesignal model is to use the disturbance approach around the working point. The variables are written as a combination of the working point value (capital letter with 0 in the index) and small disturbances (small letters with a roof $\wedge$-symbol above them) [4]

$$
\begin{aligned}
& i_{L 1}=I_{L 10}+\hat{i}_{L 1} \\
& i_{L M}=I_{M 0}+\hat{i}_{M} \\
& u_{C 1}=U_{C 10}+\hat{u}_{C 1} \\
& n=N_{0}+\hat{n} \\
& u_{1}=U_{10}+\hat{u_{1}} \\
& d=D_{0}+\hat{d} \\
& m_{L}=M_{L 0}+m_{L} .
\end{aligned}
$$

There are products between constants, products between a constant and a variable disturbance marked with $\wedge$, and products between two disturbances. The products between the constant working point values are zero (because on the left side of the equation there are only the derivatives and no added constant) and lead to the working point equations. The products between two disturbances are the only nonlinear terms. If these (small) terms are deleted, one gets the linear small signal equation [4]. The result of this equations is only the disturbance of the state variables around the working point. To get the real values, one has to add the working point value according (16).

\subsection{Small signal model}

The small signal model describes the state variables around the working point in accordance with the input variables: the disturbance of the input voltage, the disturbance of the momentum of load and the disturbance of the duty cycle as an additional input variable generated by the linearization. This new input variable is the only one which can be changed arbitrarily. The input variables, supply voltage and momentum of the load are given externally and will be the disturbance variables for the speed control.

$$
\begin{aligned}
& \frac{d}{d t}\left(\begin{array}{c}
\hat{i}_{L 1} \\
\hat{i}_{M} \\
\hat{u}_{C 1} \\
\hat{n}
\end{array}\right)= \\
& {\left[\begin{array}{cccc}
-\frac{R_{C 1}\left(1-D_{0}\right)+R_{L 1}+R_{S}}{L_{1}} & \frac{R_{C 1}\left(1-D_{0}\right)}{L_{1}} & -\frac{1-D_{0}}{L_{1}} & 0 \\
\frac{R_{C 1}\left(1-D_{0}\right)}{L_{M}} & -\frac{R_{C 1}+R_{M}}{L_{M}} & \frac{1}{L_{M}} & -\frac{C_{E}}{L_{M}} \\
\frac{1-D_{0}}{C_{1}} & -\frac{1}{C_{1}} & 0 & 0 \\
0 & \frac{C_{T}}{2 \pi J} & 0 & 0
\end{array}\right]} \\
& \left(\begin{array}{c}
\hat{i}_{L 1} \\
\hat{i}_{M} \\
\hat{u}_{C 1} \\
\hat{n}
\end{array}\right)+\left[\begin{array}{ccc}
\frac{1}{L_{1}} & 0 & \frac{R_{C 1}\left(I_{L 10}-I_{L M 0}\right)+U_{C 10}}{L_{1}} \\
-\frac{1}{L_{M}} & 0 & -\frac{R_{C 1} I_{L 10}}{L_{M}} \\
0 & 0 & -\frac{I_{L 10}}{C_{1}} \\
0 & -\frac{1}{2 \pi J} & 0
\end{array}\right]\left(\begin{array}{c}
\hat{u}_{1} \\
\hat{m}_{\text {Load }} \\
\hat{d} \\
{ }^{\prime}
\end{array}\right)
\end{aligned}
$$

\subsection{Steady state connections for the non- ideal model}

The connection of the working point values can be determined by the disturbance method, too. The influence of the parasitic elements (the losses) can be achieved by the nonlinear model (15). The variables of the equation system are the working point values $\mathrm{I}_{\mathrm{L} 10}, \mathrm{I}_{\mathrm{M} 0}, \mathrm{U}_{\mathrm{C} 10}, \mathrm{~N}_{0}$ in accordance with the steady-state input variables $\mathrm{U}_{10}, \mathrm{M}_{\mathrm{L} 0}, \mathrm{D}_{0}$. Written in matrix form one gets

$$
\begin{aligned}
& {\left[\begin{array}{cccc}
-\left[R_{C 1}\left(1-D_{0}\right)+R_{L 1}+R_{S}\right] & {\left[R_{C 1}\left(1-D_{0}\right)\right]} & \left(D_{0}-1\right) & 0 \\
{\left[R_{C 1}\left(1-D_{0}\right)\right]} & -\left(R_{C 1}+R_{M}\right) & 1 & -C_{E} \\
\left(1-D_{0}\right) & -1 & 0 & 0 \\
0 & C_{T} & 0 & 0
\end{array}\right]} \\
& \left(\begin{array}{c}
I_{L 10} \\
I_{M 0} \\
U_{C 10} \\
N_{0}
\end{array}\right)=\left[\begin{array}{cc}
-1 & 0 \\
1 & 0 \\
0 & 0 \\
0 & 1
\end{array}\right]\left(\begin{array}{c}
U_{10} \\
M_{L 0}
\end{array}\right)
\end{aligned}
$$

With the abbreviations $\mathrm{K}_{\mathrm{mn}}$ for the elements of the coefficient matrix, one can write the determinant of the matrix which is equal to

$$
D=K_{42}\left|\begin{array}{ccc}
K_{11} & K_{13} & 0 \\
K_{21} & K_{23} & K_{24} \\
K_{31} & 0 & 0
\end{array}\right|=K_{13} K_{24} K_{31} K_{42}=C_{E} C_{T}\left(1-D_{0}\right)^{2}
$$

The numerator of the connection between the input voltage and the speed can be calculated according to 


$$
N_{N 0 U 10}=K_{42}\left(K_{13} K_{31}+K_{31} K_{23}\right)=C_{T}\left(1-D_{0}\right) D_{0}
$$

this leads to the connection

$$
N_{0}=\frac{N_{N 0 U 10}}{D}=\frac{D_{0}}{C_{E}\left(1-D_{0}\right)} U_{10} .
$$

This equation describes the drive with no load. The momentum of the load leads to a current in the machine, which leads to a voltage drop across the armature resistor and to a reduction of the speed.

The numerator for the influence of the load momentum to the speed can be calculated according to

$N_{\text {NOMLO }}=K_{31}\left(K_{12} K_{23}-K_{22} K_{13}\right)-K_{32}\left(K_{11} K_{23}-K_{21} K_{13}\right)$.

This leads to

$$
\frac{N_{0}}{M_{L 0}}=-\frac{1}{C_{E} C_{T}}\left[R_{M}+\frac{R_{L 1}+R_{S}}{\left(1-D_{0}\right)^{2}}+\frac{R_{C 1} D_{0}}{\left(1-D_{0}\right)}\right] .
$$

For $R_{M}>>R_{L 1}+R_{S}$ and $R_{M}>>R_{C 1}$ one gets

$$
\frac{N_{0}}{M_{L 0}}=-\frac{R_{M}}{C_{E} C_{T}} .
$$

Since the system is linear, one can now write for the stationary speed

$$
N_{0}=\frac{D_{0}}{C_{E}\left(1-D_{0}\right)} U_{10}-\frac{R_{M}}{C_{E} C_{T}} M_{L 0} .
$$

The speed is directly proportional to the input voltage reduced by the influence of the momentum of the load.

\section{$4 \quad$ Idealized Model}

The losses in the converter are low (the parasitic resistors are small) compared to the loss in the machine (the armature resistor is large compared to the parasitic resistors of the converter). In the idealized model we skip the parasitic losses of the converter and include only the resistor of the machine. The idealization concerns only the converter and not the machine.

\subsection{Idealized nonlinear model}

The losses of the converter elements are small compared to the resistor of the machine. The efficiency of the converter is determined by these ohmic losses. Especially in the low voltage range active switches with very low on-resistance can be purchased. High switching frequencies also reduce the size of the converter coil and of the converter capacitor. The value of $\mathrm{L}_{1}$ and $\mathrm{C}_{1}$ are indirectly proportional to the frequency (cf. 35, 37). The smaller the inductor the smaller is the parasitic resistor and the smaller are the losses of the coil. The switching losses can be reduced by snubber circuits or by using ZVS (zero voltage switching). Omitting now all losses in (15) except the armature resistor leads to

$$
\begin{aligned}
\frac{d}{d t}\left(\begin{array}{c}
i_{L 1} \\
i_{M} \\
u_{C 1} \\
n
\end{array}\right) & =\left[\begin{array}{cccc}
0 & 0 & -\frac{1-d}{L_{1}} & 0 \\
0 & -\frac{R_{M}}{L_{M}} & \frac{1}{L_{M}} & -\frac{C_{E}}{L_{M}} \\
\frac{1-d}{C_{1}} & -\frac{1}{C_{1}} & 0 & 0 \\
0 & \frac{C_{T}}{2 \pi J} & 0 & 0
\end{array}\right]\left(\begin{array}{c}
i_{L 1} \\
i_{M} \\
u_{C 1} \\
n
\end{array}\right)+ \\
& +\left[\begin{array}{cc}
\frac{1}{L_{1}} & 0 \\
-\frac{1}{L_{M}} & 0 \\
0 & 0 \\
0 & -\frac{1}{2 \pi J}
\end{array}\right]\left(\begin{array}{c}
u_{1} \\
m_{\text {Load }}
\end{array}\right]
\end{aligned}
$$

\subsection{Idealized linear model}

Using (16) leads to

$$
\begin{aligned}
\frac{d}{d t}\left(\begin{array}{c}
\hat{i}_{L 1} \\
\hat{i}_{M} \\
\hat{u}_{C 1} \\
\hat{n}
\end{array}\right) & =\left[\begin{array}{cccc}
0 & 0 & -\frac{1-D_{0}}{L_{1}} & 0 \\
0 & -\frac{R_{M}}{L_{M}} & \frac{1}{L_{M}} & -\frac{C_{E}}{L_{M}} \\
\frac{1-D_{0}}{C_{1}} & -\frac{1}{C_{1}} & 0 & 0 \\
0 & \frac{C_{T}}{2 \pi J} & 0 & 0
\end{array}\right]\left(\begin{array}{c}
\hat{i}_{L 1} \\
\hat{i}_{M} \\
\hat{u}_{C 1} \\
\hat{n}
\end{array}\right) . \\
& +\left[\begin{array}{ccc}
\frac{1}{L_{1}} & 0 & \frac{U_{C 10}}{L_{1}} \\
-\frac{1}{L_{M}} & 0 & 0 \\
0 & 0 & -\frac{I_{L 10}}{C_{1}} \\
0 & -\frac{1}{2 \pi J} & 0
\end{array}\right]\left(\begin{array}{c}
\hat{u}_{1} \\
\hat{m}_{\text {Load }} \\
\hat{d}
\end{array}\right)
\end{aligned}
$$

This equation describes the disturbances around the working point for small deviations from the steadystate value.

\subsection{Stationary connections for the working point}

The values of the working point and their connections can be found again with the disturbance method by summing up all constant values after substituting (16) into (27).

One gets

$$
\begin{array}{ll}
\text { a } & \left(1-D_{0}\right) U_{C 10}=U_{10} \\
& U_{C 10}=\frac{1}{1-D_{0}} U_{10} \\
\mathrm{~b} & -R_{M} I_{M 0}+U_{C 10}-C_{E} n_{0}=U_{10} \\
& n_{0}=\frac{1}{C_{E}}\left(\frac{D_{0}}{1-D_{0}} U_{10}-R_{M} I_{M 0}\right) \\
& \left(1-D_{0}\right) I_{L 10}=I_{M 0} \\
\mathrm{c} & C_{T} I_{M 0}=M_{\text {Load } 0} .
\end{array}
$$




\section{$5 \quad$ Transfer functions}

Starting from the idealized linear state-space model (27), one can calculate the transfer functions with the abbreviations $A_{m n}$ for the elements of the state matrix and $\mathrm{B}_{\mathrm{mn}}$ for the elements of the input matrix, with $m$ as the number of the lines and $n$ as the number of the columns. Laplace transformation leads to

$$
\begin{aligned}
& {\left[\begin{array}{cccc}
s & 0 & -A_{13} & 0 \\
0 & s-A_{22} & -A_{23} & -A_{24} \\
-A_{31} & -A_{32} & s & 0 \\
0 & -A_{42} & 0 & s
\end{array}\right]\left(\begin{array}{c}
I_{L 1}(s) \\
I_{M}(s) \\
U_{C 1}(s) \\
N(s)
\end{array}\right)=} \\
& =\left[\begin{array}{ccc}
B_{11} & 0 & B_{13} \\
B_{21} & 0 & 0 \\
0 & 0 & B_{33} \\
0 & B_{42} & 0
\end{array}\right]\left(\begin{array}{c}
U_{1}(s) \\
M_{\text {Load }}(s) \\
D(s)
\end{array}\right)
\end{aligned}
$$

With four variables and three known variables twelve transfer functions can be calculated. The most important ones are those which describe the influence of the input variables on the speed. The denominator is the same for all transfer functions

$$
\begin{aligned}
D= & s^{4}-s^{3} A_{22}-s^{2}\left(A_{24} A_{42}+A_{13} A_{31}+A_{23} A_{32}\right)+ \\
& +s A_{13} A_{22} A_{31}+A_{13} A_{24} A_{31} A_{42}
\end{aligned}
$$

For the numerator of the function between speed and duty cycle one gets

$$
N_{-} N D=s A_{23} B_{33} A_{42}+A_{23} A_{31} B_{13}
$$

The substitution of the coefficients shows that the system is a non-phase-minimum one. For the numerator of the transfer function between speed and momentum of load one gets

$N_{-} N M=B_{42}\left(s^{3}-s^{2} A_{22}-s\left(A_{13} A_{31}+A_{23} A_{32}\right)+A_{13} A_{22} A_{31}\right)$

For the numerator of the transfer function between speed and input voltage one gets

$$
N \_N U=A_{42}\left[s^{2}\left(A_{23}+B_{21}\right)-A_{13} A_{31} B_{21}+A_{23} A_{31} B_{11}\right] \text {. }
$$

$\mathrm{A}_{23}$ and $\mathrm{B}_{21}$ have the same value, but a different sign. Therefore, the denominator has no quadratic term. The entire three transfer functions are given in the appendix.

The data for the machine are:

$$
\begin{aligned}
& \mathrm{R}_{\mathrm{M}}=0.4 \Omega, \mathrm{L}_{\mathrm{M}}=380 \mu \mathrm{H}, \mathrm{C}_{\mathrm{E}}=0.64 \mathrm{Vs}, \\
& \mathrm{C}_{\mathrm{T}}=0.076 \mathrm{Nm} / \mathrm{A}, \mathrm{J}=0.007 \mathrm{kgm} 2
\end{aligned}
$$

and the parameters of the converter are:

$$
\mathrm{L}_{1}=60 \mu \mathrm{H}, \mathrm{C}_{1}=330 \mu \mathrm{F} \text {. }
$$

The chosen work point values are

$$
\begin{aligned}
& \mathrm{U}_{10}=24 \mathrm{~V}, \mathrm{I}_{\mathrm{M} 0}=10 \mathrm{~A}, \mathrm{D}_{0}=0.5, \\
& \mathrm{U}_{\mathrm{C} 10}=48 \mathrm{~V}, \mathrm{~N}_{0}=31 \mathrm{rps}, \mathrm{I}_{\mathrm{L} 10}=20 \mathrm{~A} .
\end{aligned}
$$

Fig. 13 shows the connection between speed and duty cycle. This is the frequency plot of the system to be controlled.

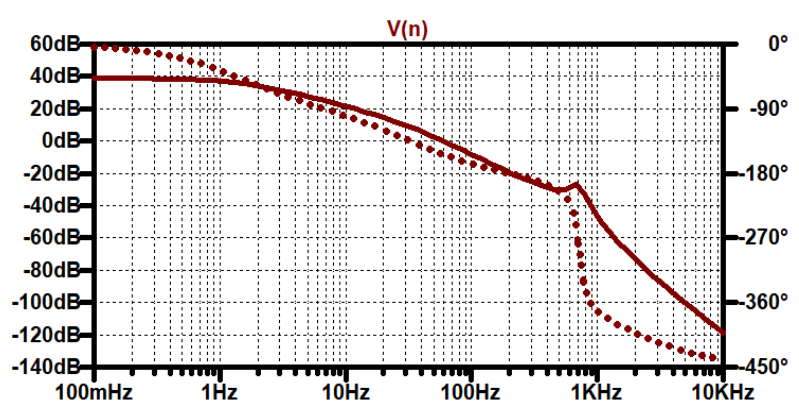

Fig. 13. Bode plot between speed and duty cycle

The system is a fourth order one and is, due to the zero in the right-half plane, a non-minimum-phase system and therefore the phase goes to minus 450 degrees.

In Fig. 14 the frequency response between the momentum of the load and the speed is depicted.

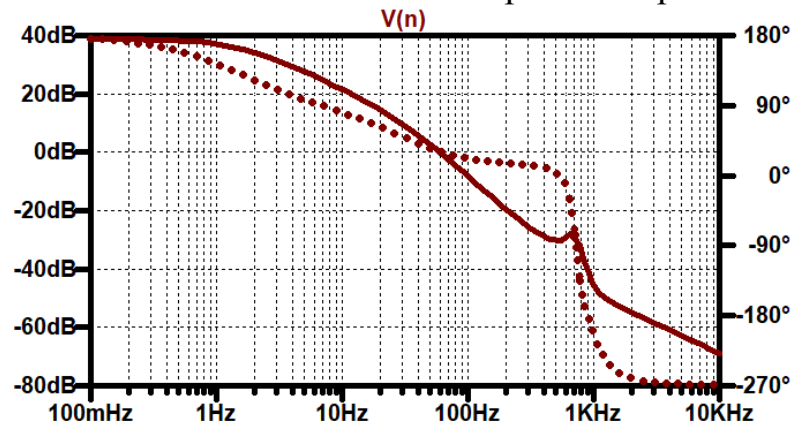

Fig. 14. Bode plot between speed and momentum of the load

Due to the negative sign of the transfer function, the phase plot starts from plus $180^{\circ}$ and shifts at high frequencies by $-450^{\circ}$ (the denominator shifts by minus $360^{\circ}$, the numerator by a further minus $90^{\circ}$ due to the right half-plane zero), leading to a phase shift of $-270^{\circ}$ at high frequencies.

Fig. 15 shows the Bode plot of the speed in dependence on the supply voltage.

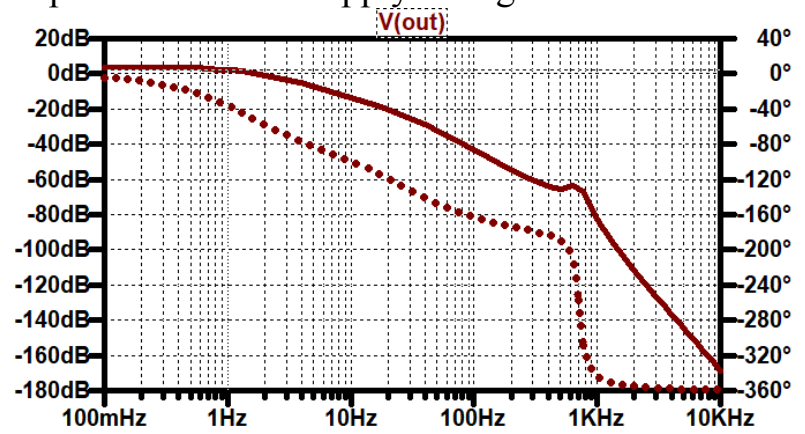

Fig. 15. Bode plot between speed and input voltage

This Bode plot shows a minimum-phase system. The dynamics of the system are dominated by the mechanical time constant caused by the momentum of inertia of the drive. To get the real dynamics of the drive (and not only of the machine with the converter), one has to add the momentum of the load to the value of that of the machine. The 
equations do not change, only the value of $\mathrm{J}$. The equations are given in the appendix. The slowest time constant is caused by the machine, the electrical time constant of the machine is faster and the fastest time constant is that of the converter.

\section{Dimensioning of the converter}

The output capacitor of a normal Buck-Boost and the capacitor between input and output of the modified Buck-Boost have the same value. During the on-time of the active switch, the capacitor is discharged by the load current (Fig. 4). The voltage decreases by the value

$$
\Delta u_{C 1}=\frac{1}{C_{1}} \int_{0}^{d \cdot T} I_{M 0} \cdot d t .
$$

With the help of the voltage transformation ratio (1) and using $\mathrm{U}_{\mathrm{M}}$ for the voltage across the armatures, one gets for the capacitor

$$
C_{1}=\frac{I_{M 0}}{\Delta u_{C 1}} \cdot \frac{U_{M}}{\left(U_{1}+U_{M}\right) \cdot f} .
$$

The inductor $\mathrm{L}_{1}$ can be designed for a desired current ripple $\Delta \mathrm{I}_{\mathrm{L} 1}$ according to

$$
L_{1}=\frac{U_{1} d T}{\Delta I_{L 1}} .
$$

By replacing $d$ again with the help of (1) and using the frequency instead of the time period, one gets

$$
L_{1}=\frac{U_{1} U_{M}}{\left(U_{1}+U_{M}\right) \Delta I_{L 1} f} .
$$

The maximum voltage across the semiconductors and the capacitor $\mathrm{C}_{1}$ is the sum of the input voltage and the armature voltage of the machine. To choose these devices an appropriate security factor depending on the application must be applied.

The voltage stress of the semiconductor devices is equal to the voltage across $C_{1}$ and therefore equal to the sum of input and output voltages. For industrial electronics applications one would choose a security factor of 1.6 to 2 to get the necessary blocking voltage of the devices.

To reduce the switching losses, zero voltage switching (ZVS) can be achieved by connecting small capacitors in parallel to the active switches and control the switches accordingly (c.f. e.g. [5]). When $S_{1}$ turns off, the current commutates into these small capacitors, the one in parallel to $S_{1}$ is charged and the other, which is in parallel to $S_{2}$, is discharged. When the voltage across $\mathrm{S}_{2}$ reaches zero, the (body) diode in parallel to $\mathrm{S}_{2}$ turns on. Now one can turn on $\mathrm{S}_{2}$ with ZVS to shunt the diode and to reduce the losses. In this concept a special control should be used and the switching frequency will not be constant anymore. The value of $L_{1}$ can be decreased also because a higher current ripple is now possible.

\section{Simulation}

Fig. 15 shows the current through the devices of the converter, when energy is transferred from the source to the machine. The converter is working at the border of the step-down mode. Therefore, the input current (turquoise) is nearly equal to the current through the machine (green). The ripple of the input current is the same as the one through the inductor $\mathrm{L}_{1}$ and can be adjusted by (37). The signals are similar to those which were constructed in section 1 . Only another duty cycle (50\%) was used.

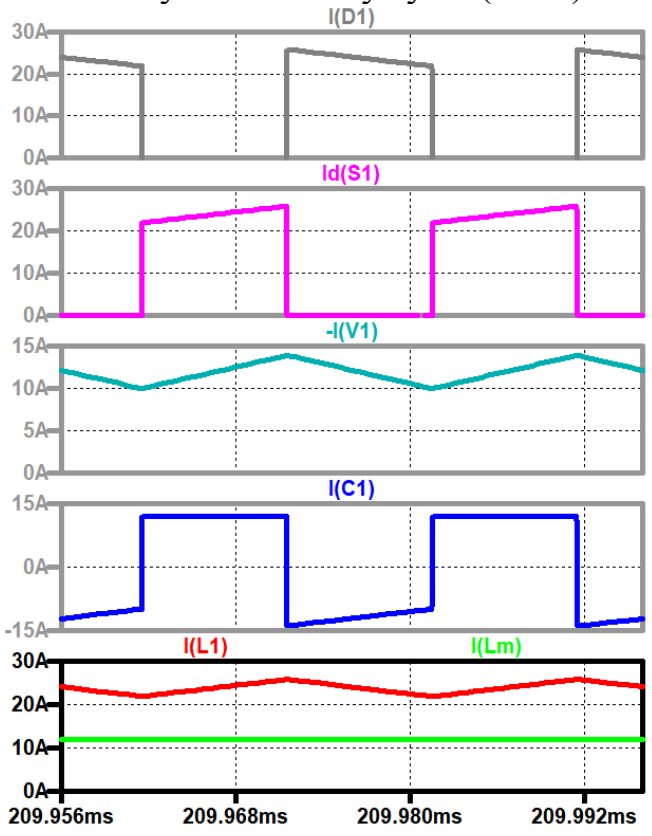

Fig. 15. up to down the currents through: the diode, the transistor, the input, the capacitor $\mathrm{C}_{1}$ and the inductors of the converter and of the machine

The machine is, caused by the momentum of inertia, much slower than the converter. The control of the drive can be done by a sliding mode or bang-bang controller for the current through $\mathrm{L}_{1}$ and by a slow PI-controller as an outer loop for the speed.

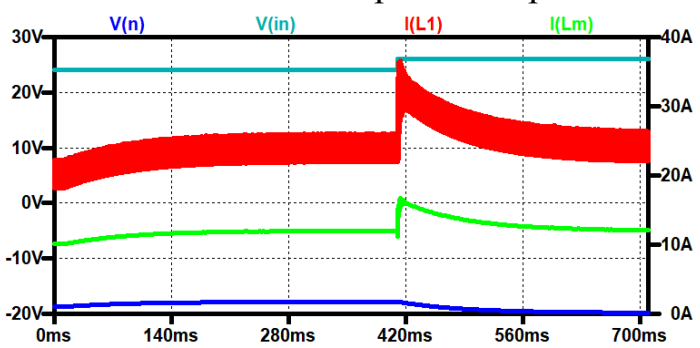

Fig. 16. Current through $\mathrm{L}_{1}$ (red), $\mathrm{L}_{\mathrm{M}}$ (green), speed (n), input voltage (turquoise)

Fig. 16 shows a momentum of the load step at the beginning; the current increases and the speed decreases (blue, keep in mind that the system is an 
inverting one, therefore the blue curve increases when the speed decreases). The speed is not directly shown, the blue curve is the source voltage of the machine which is proportional to the speed. The red curve shows the current through the coil of the converter. It is a band, caused by the current ripple. The current through the machine (green) has practically no ripple, as predicted in the theoretical analyses. Later (at $400 \mathrm{~ms}$ ) an input voltage step occurs, now the speed increases again and one can see the transient in the currents.

\section{Conclusion}

After a basic analyzes of the modified Buck-Boost converter with integrated output filter in section 1 , the application of the converter as a motor control unit is discussed. To achieve two-quadrant operation, two active switches connected as a halfbridge have to be used instead of one active and one passive switch. In the paper all necessary aspects for the application of this converter (model, dimensioning, transfer functions) were treated.

This converter has a very simple structure and has several advantages when controlling a DC machine:

- Lower and higher voltages can be applied to the machine

- Driving and braking (two-quadrant operation) for one direction are possible

- The input current is continuous

- Only a small converter capacitor is necessary

- There is always a nearly constant voltage across the machine

- Push-pull control of the switches leads always to continuous inductor current

Transfer function between speed and duty cycle

\section{APPENDIX}

$$
\frac{N(s)}{D(s)}=\frac{C_{T}}{2 \pi J} \frac{-s \frac{I_{L 10}}{C_{1} L_{M}}+\frac{\left(1-D_{0}\right) U_{C 10}}{C_{1} L_{1} L_{M}}}{s^{4}+s^{3} \frac{R_{M}}{L_{M}}+s^{2}\left(\frac{C_{E} C_{T}}{2 \pi J L_{M}}+\frac{\left(1-D_{0}\right)^{2}}{C_{1} L_{1}}+\frac{1}{C_{1} L_{M}}\right)+s \frac{\left(1-D_{0}\right)^{2} R_{M}}{C_{1} L_{1} L_{M}}+\frac{C_{E} C_{T}\left(1-D_{0}\right)^{2}}{2 \pi J C_{1} L_{1} L_{M}}}
$$

Transfer function between speed and momentum of the load (with the same denominator)

$$
\frac{N(s)}{M_{L}(s)}=\frac{-\frac{1}{2 \pi J}\left[s^{3}+s^{2} \frac{R_{M}}{L_{M}}+s\left(\frac{\left(1-D_{0}\right)^{2}}{L_{1} C_{1}}+\frac{1}{C_{1} L_{M}}\right)+\frac{\left(1-D_{0}\right)^{2} R_{M}}{C_{1} L_{1} L_{M}}\right]}{N(s)}
$$

Transfer function between speed and input voltage (with the same denominator)

$$
\frac{N(s)}{U_{1}(s)}=\frac{\frac{C_{T}}{2 \pi J} \frac{\left(1-D_{0}\right) D_{0}}{C_{1} L_{1} L_{M}}}{N(s)}
$$




\section{Creative Commons Attribution License 4.0} (Attribution 4.0 International, CC BY 4.0)

This article is published under the terms of the Creative Commons Attribution License 4.0

https://creativecommons.org/licenses/by/4.0/deed.en US 